\title{
Correlations Between Linear and Nonlinear Functions of Discrete Random Variables
}

\author{
Nanda Pandharikar* \\ Associate Professor, Department of Mathematics and Statistics, Sydenham College of Commerce and \\ Economics, Mumbai, India \\ *Corresponding author: shrinathsdp@gmail.com
}

\begin{abstract}
In this article correlation between simple linear and nonlinear functions of two random variables have been discussed. The study is carried out for two bivariate geometric distributions.

Keywords: Bivariate distribution, Coefficient of correlation, Geometric distribution.
\end{abstract}

\section{Introduction}

The study of correlation is carried out for two types of bivariate geometric distributions of the type given below:

I. Bivariate distributions having single parameter $\theta$. We refer to this as bivariate distribution- I.

II. Bivariate distribution having two parameters. We refer to this as bivariate distribution-II.

Now we consider the first type of distribution.

\section{Bivariate Distribution - I}

Here we consider the bivariate distribution with the joint probability mass function of $(\mathrm{X}, \mathrm{Y})$ is given by

$$
\begin{array}{rl}
p(x, y)=\left(\begin{array}{c}
x+y \\
x
\end{array}\right) \theta^{y+2}(1-\theta)^{x+y} & 0 \leq x, y \\
=0 & \text { otherwise }
\end{array}
$$

Where $\theta$ is a parameter of the distribution such that $0<\theta<1$.

A. Marginal probability function of $X$

$$
\begin{aligned}
& p(X=x)=p(x) \\
= & \sum_{y=0}^{\infty} p(x, y) \\
= & \sum_{y=0}^{\infty}\left(\begin{array}{c}
x+y \\
x
\end{array}\right) \theta^{y+2}(1-\theta)^{x+y} \\
= & \frac{\theta^{2}}{\left(1-\theta+\theta^{2}\right)}\left(\frac{1-\theta}{1-\theta+\theta^{2}}\right)^{x} \quad x=0,1,2 \ldots
\end{aligned}
$$

Which is a geometric distribution with parameter $\left[\theta^{2} /\left(1-\theta+\theta^{2}\right)\right]$.

\section{B. Marginal probability function of $Y$}

Here we derive marginal probability function of $\mathrm{Y}$ and its four moments.

The marginal probability function of $\mathrm{Y}$ is given by,

$$
\begin{aligned}
p(Y=y) & =p(y) \\
& =\sum_{x=0}^{\infty} p(x, y) \\
& =\sum_{x=0}^{\infty}\left(\begin{array}{c}
x+y \\
x
\end{array}\right) \theta^{y+2}(1-\theta)^{x+y} \\
& =\theta(1-\theta)^{y}, \quad y=0,1,2 \ldots
\end{aligned}
$$

Which is Geometric distribution with parameter $\theta$.

Here we consider simple linear functions as $\mathrm{X}$ and $\mathrm{Y}$ and nonlinear function as $\mathrm{X} 2$ and $\mathrm{Y} 2$.

Correlation between $\mathrm{X}$ and $\mathrm{Y}$ can be considered as correlation between linear functions of $\mathrm{X}$ and $\mathrm{Y}$.

Similarly, correlation between $X^{2}$ and $Y$ or between $X$ and $\mathrm{Y}^{2}$ means correlation between linear and nonlinear functions of

Table 1

Bivariate distribution - I

\begin{tabular}{|c|c|}
\hline $\begin{array}{c}\text { Pair of } \\
\text { Random } \\
\text { variables }\end{array}$ & Expression for Coefficient of Correlation \\
\hline$(\mathrm{X}, \mathrm{Y})$ & $\frac{(1-\theta)}{\sqrt{1-\theta+\theta^{2}}}$ \\
\hline$\left(\mathrm{X}^{2}, \mathrm{Y}\right)$ & $\frac{(1-\theta)}{\sqrt{1-\theta+\theta^{2}}} \sqrt{\sqrt{\left[2(1-\theta)+\theta^{2}\right]} \sqrt{\left[10(1-\theta)+\theta^{2}\right]}}$ \\
\hline$(\mathrm{X}, \mathrm{Y} 2)$ & $\frac{(1-\theta)}{\sqrt{\left(1-\theta+\theta^{2}\right)}}\left[\frac{(4-3 \theta)}{\sqrt{(2-\theta)(10-9 \theta)}}\right]$ \\
\hline$\left(\mathrm{X}^{2}, \mathrm{Y}^{2}\right)$ & $\frac{(1-\theta)}{\sqrt{1-\theta+\theta^{2}}}\left[\frac{\left[20\left(1-\theta^{2}\right)-3 \theta\left(12+\theta^{2}\right)\right]}{\sqrt{\left[2(1-\theta)+\theta^{2}\right](2-\theta)\left[10(1-\theta)+\theta^{2}\right](10-9 \theta)}}\right]$ \\
\hline
\end{tabular}


$\mathrm{X}$ and Y. For above two distributions, four correlation coefficients are derived and given in table 1.

Now we consider second type of distribution.

\section{Bivariate Distribution - II}

Random variable $(\mathrm{X}, \mathrm{Y})$ belonging to the class of discrete bivariate distributions of type II has joint probability mass function is given by,

$$
\begin{gathered}
p(x, y)=\left(\begin{array}{c}
x+y \\
y
\end{array}\right) \theta_{1}^{x} \theta_{2}^{y} \theta_{0}, \quad x, y=0,1,2, \ldots \\
\theta_{0}=1-\theta_{1}-\theta_{2} \\
0<\theta_{i}<\theta_{1}+\theta_{2}<1, \quad i=1,2 \\
=0 \quad \text { otherwise }
\end{gathered}
$$

Now we discuss the properties of random variables $\mathrm{X}$ and $\mathrm{Y}$ to study their behavior separately.

\section{A. Marginal Distribution of $X$}

First we derive marginal probability function of $\mathrm{X}$. It is given by,

$$
\begin{aligned}
p[X=x] & =p(x) \\
& =\theta_{0} \theta_{1}^{x} \sum_{y=0}^{\infty}\left(\begin{array}{c}
x+y \\
y
\end{array}\right) \theta_{2}^{y} \\
& =\frac{\theta_{0}}{\left(1-\theta_{2}\right)}\left(\frac{\theta_{1}}{1-\theta_{2}}\right)^{x} \quad x=0,1,2, \ldots
\end{aligned}
$$

We note that $\mathrm{X}$ has Geometric distribution with parameter,

$$
\frac{\theta_{0}}{\left(1-\theta_{2}\right)}
$$

where $\theta_{0}=1-\theta_{1}-\theta_{2}$.

Now we derive marginal distribution of $\mathrm{Y}$.

\section{B. Marginal Distribution of $Y$}

$$
\begin{aligned}
p[Y=y] & =p(y) \\
& =\sum_{x=0}^{\infty} p(x, y) \\
& =\theta_{0} \theta_{2}{ }^{y} \sum_{x=0}^{\infty}\left(\begin{array}{c}
x+y \\
x
\end{array}\right) \theta_{1}^{x} \\
& =\frac{\theta_{0}}{\left(1-\theta_{1}\right)}\left(\frac{\theta_{2}}{1-\theta_{1}}\right)^{y} \quad y=0,1,2, \ldots
\end{aligned}
$$

Where $\theta_{0}=1-\theta_{1}-\theta_{2}$.

Random Variable Y also has Geometric distribution with parameter,

$$
\frac{\theta_{0}}{\left(1-\theta_{1}\right)}
$$

Now coefficients of correlation between the pairs of random variables such as $(\mathrm{X}, \mathrm{Y}),\left(\mathrm{X}^{2}, \mathrm{Y}\right),\left(\mathrm{X}, \mathrm{Y}^{2}\right)$ and $\left(\mathrm{X}^{2}, \mathrm{Y}^{2}\right)$ are derived

\begin{tabular}{|c|c|c|c|}
\hline \multicolumn{2}{|c|}{$\theta_{1}=0.4$} & & \\
\hline$\theta$ & $\rho\left(X^{2}, Y\right)$ & $\rho\left(X, Y^{2}\right)$ & $\rho\left(X^{2}, Y\right) /\left(X, Y^{2}\right)$ \\
\hline 0.1 & 0.239 & 0.2363 & 1.0114 \\
\hline 0.2 & 0.3535 & 0.3558 & 1.00630 \\
\hline 0.3 & 0.4652 & 0.4669 & 0.99635 \\
\hline 0.4 & 0.5855 & 0.5855 & 1. \\
\hline 0.5 & 0.7239 & 0.7225 & 1.001937 \\
\hline 0.6 & 0.8944 & 0.8944 & 1. \\
\hline
\end{tabular}
for this distribution and given in table 2 .

Table 2

Bivariate distribution - II

\begin{tabular}{|c|c|}
\hline $\begin{array}{c}\text { Pair of } \\
\text { Random } \\
\text { variables }\end{array}$ & Expression for correlation coefficient \\
\hline$(\mathrm{X}, \mathrm{Y})$ & $\sqrt{\frac{\theta_{1}}{\left(\theta_{0}+\theta_{2}\right)} \frac{\theta_{2}}{\left(\theta_{0}+\theta_{1}\right)}}$ \\
\hline$\left(\mathrm{X}^{2}, \mathrm{Y}\right)$ & $\left(4 \theta_{1}+\theta_{0}\right) \sqrt{\frac{\theta_{1} \theta_{2}}{\left(\theta_{0}+\theta_{2}\right)\left(\theta_{0}+\theta_{1}\right)\left(\theta_{0}+2 \theta_{1}\right)\left(\theta_{0}+10 \theta_{1}\right)}}$ \\
\hline$\left(\mathrm{X}, \mathrm{Y}^{2}\right)$ & $\left(4 \theta_{2}+\theta_{0}\right) \sqrt{\frac{\theta_{1} \theta_{2}}{\left(\theta_{0}+\theta_{1}\right)\left(\theta_{0}+\theta_{2}\right)\left(\theta_{0}+2 \theta_{2}\right)\left(\theta_{0}+10 \theta_{2}\right)}}$ \\
\hline$\left(\mathrm{X}^{2}, \mathrm{Y}^{2}\right)$ & $\frac{\mu_{22}^{\prime}-\mu_{20}^{\prime} \mu_{02}^{\prime}}{\sqrt{\left(\mu_{40}^{\prime}-\mu_{20}^{\prime}\right)} \sqrt{\left(\mu_{04}^{\prime}-\mu_{02}^{\prime}{ }^{2}\right)}}$ \\
\hline
\end{tabular}

Where the raw moments in the above table are complicated so it was avoided.

To study the behavior of these correlation coefficients $\rho\left(X^{2}, Y\right)$ and $\rho\left(X, Y^{2}\right)$, their ratio is calculated for two values of $\theta_{1}=0.3$ and $\theta_{2}=0.4$.

Following tables show the coefficient of correlation between $\mathrm{X}^{2}$ and $\mathrm{Y}, \mathrm{X}$ and $\mathrm{Y}^{2}$ and their ratio for $\theta_{1}=0.3$ and $\theta_{2}=0.4$.

\begin{tabular}{|c|l|l|l|}
\multicolumn{3}{c}{$\begin{array}{c}\text { Table } 3 \\
\theta_{1}=0.3\end{array}$} \\
\hline $\boldsymbol{\theta}$ & $\boldsymbol{\rho}\left(\mathbf{X}^{\mathbf{2}}, \mathbf{Y}\right)$ & $\boldsymbol{\rho}\left(\mathbf{X}, \mathbf{Y}^{\mathbf{2}}\right)$ & $\boldsymbol{\rho}\left(\mathbf{X}^{\mathbf{2}}, \mathbf{Y}\right) /\left(\mathbf{X}, \mathbf{Y}^{\mathbf{2}}\right)$ \\
\hline 0.1 & 0.1889 & 0.1928 & 0.979771 \\
\hline 0.2 & 0.2835 & 0.2836 & 0.979426 \\
\hline 0.3 & 0.3718 & 0.3718 & 1 \\
\hline 0.4 & 0.4652 & 0.4669 & 0.989968 \\
\hline 0.5 & 0.5728 & 0.5765 & 0.9935 \\
\hline 0.6 & 0.7075 & 0.7118 & 0.9839839 \\
\hline 0.7 & 0.8944 & 0.8944 & 1 \\
\hline
\end{tabular}

\section{Conclusion}

Correlation plays very important role in studying relationship 
between the variables. Study of correlation further extended to study the correlation for simple functions. So bivariate geometric distributions are considered for this purpose. It is observed that the ratio $\rho\left(\mathrm{X}^{2}, \mathrm{Y}\right) /\left(\mathrm{X}, \mathrm{Y}^{2}\right)$ is nearly equal to 1 .

\section{References}

[1] A. Mood, F. Graybill, and D. Boes, "Introduction to the theory of Statistics," Third Edition McGraw-Hill international book company, 1913.
[2] P. Mukhopadhyay, "Mathematical Statistics," New Central Book Agency (P) Ltd.1996.

[3] A. G. Phatak, and M. Sreehari, "Some Characterizations of a bivariate geometric distribution," in Journal of Indian Statistical Association, vol. 19, 141-146, 1981.

[4] C. R. Rao, "Linear Statistical inference and its Applications," Second Edition, Wiley, New York, 1973

[5] V. K. Rohatgi, "An introduction to probability theory and mathematical Statistics," Wiley Eastern Limited., 1993.

[6] M. Sreehari, "Characterizations via conditional distributions," in Journal of Indian Statistical Association, vol. 43, 77-93, 2005.

[7] M. Sreehari, "Bivariate Geometric Distributions Revisited," in Sci. Lett. Vol. 27, no. 1-2, pp. 13-26, 2003. 